\title{
Institutionelle Dimensionen inklusiver Schulbildung - Herausforderungen der UN-Behindertenrechtskonvention für Deutschland, Island und Schweden im Vergleich
}

\author{
Julia Biermann • Justin J.W. Powell
}

Online publiziert: 23. Oktober 2014

(C) Springer Fachmedien Wiesbaden 2014

Zusammenfassung Die menschenrechtsbasierte Orientierung der UN-Behindertenrechtskonvention, nach welcher inklusive Bildung als Recht Aller und Segregation auf Basis von Behinderungen als Diskriminierung gilt, stellt weltweit eine besondere Herausforderung für institutionalisierte sonderpädagogische Fördersysteme dar. In diesem Beitrag werden entlang der rechtlichen, normativen und kulturell-kognitiven Dimension inklusiver Bildung Deutschland, Island und Schweden verglichen. Der Vergleich verdeutlicht wichtige Unterschiede in diesen drei institutionalisierten Dimensionen von sonderpädagogischem Förderbedarf - Bildungsund Behinderungsparadigmen, schulischen Organisationsformen sowie Rechten und Ressourcen - die inklusive Bildung fördern oder behindern. Kontrastiert werden die Länder im Hinblick auf die Frage: Wie wird die Förderung von als förderbedürftig wahrgenommenen Schülerinnen und Schülern in diesen drei nationalen Kontexten gestaltet? Aus dieser Analyse zweier nordischer Länder, welche zu den inklusivsten weltweit zählen, werden Hinweise auf die Verwirklichung inklusiver Schulbildung in Deutschland, einem der segregiertesten Bildungssysteme Europas, abgeleitet. Die Untersuchung zeigte entscheidende Differenzen im Hinblick auf das Verhältnis von Klassifizierung und Lernortzuweisungen.

Schlüsselwörter Sonderpädagogische Förderung · Inklusive Bildung · Institution · Ländervergleich

Prof. Dr. J.J.W. Powell ( $\bowtie)$

Institute of Education \& Society, Universität Luxemburg,

Route de Diekirch,

7220 Walferdange, Luxemburg

E-Mail: justin.powell@uni.lu

J. Biermann

Arbeitsgebiet Inklusive Pädagogik, Universität Bremen,

Bibliothekstraße/GW2,

28359 Bremen, Deutschland

E-Mail: julia.biermann@posteo.de 


\title{
Institutional dimensions of inclusive schooling: comparing the challenge of the UN Convention on the Rights of Persons with Disabilities in Germany, Iceland and Sweden
}

\begin{abstract}
The human rights-based orientation embedded in the UN Convention on the Rights of Persons with Disabilities, which mandates inclusive education for all students and counts segregation as discrimination, poses fundamental challenges to institutionalized (special) education systems. However, it does so to different extents, with the Nordic countries having the most inclusive systems worldwide. This analysis contrasts the challenges and opportunities regarding the institutional transformation of special education and inclusive education in Germany, Iceland and Sweden. We address the questions: How do these countries provide educational supports for students considered to have special educational needs? What perspectives can be derived for the implementation of inclusive education, especially for Germany, which is still among the most segregated systems in Europe, from such comparative analysis? The study reveals key differences in three institutional dimensions that hinder or enable inclusive education - educational ideals and disability paradigms, organizational forms, and regulations.
\end{abstract}

Keywords Special education · Inclusive schooling $\cdot$ Institution · Cross-country comparison

\section{Die globale Diffusion des Menschenrechts auf Bildung zwischen sonderpädagogischer Förderung und inklusiver Bildung}

Mit dem Übereinkommen über die Rechte von Menschen mit Behinderungen, kurz: UN-Behindertenrechtskonvention (UN-BRK), verabschiedet von der UN-Generalversammlung als Resolution 61/106 am 13.12.2006, wird die Entwicklung inklusiver Bildungssysteme zur internationalen Norm und staatlichen Verpflichtung. Der mit der Ratifizierung der UN-BRK geschlossene völkerrechtliche Vertrag enthält für alle 147 Staaten (United Nations 2014a) einen staatlichen Gesetzgebungsauftrag, welcher in Bezug auf Bildung in Artikel 24 verankert ist. Bereits während der Verhandlungen zur UN-BRK wurde der auf Bildung bezogene Artikel am kontroversesten diskutiert. Konfliktpunkt war vor allem die Frage, ob es um die Wahlmöglichkeit, das Recht oder die Abschaffung von Sonderbeschulung gehen sollte. ${ }^{1}$ Von expliziten Formulierungen wurde schließlich abgesehen und allgemein das Menschenrecht auf inklusive Bildung verankert (Degener 2009, S. 214). Dieser Kompromiss resultiert in einer Offenheit, welche sich ebenfalls in der nicht abschließenden Definition von Behinderung wiederfindet und damit den relationalen, kontextgebundenen Charakter beider Konzepte zum Ausdruck bringt. Dies resultiert in vielfältigen, oft kontroversen Perspektiven auf schulische Inklusion, welche sich vor allem durch ihren normativen Charakter auszeichnen. Im Hinblick auf Fragen der Umsetzung kann zwischen drei institutionellen Dimensionen unterschieden werden - Bildungsidealen und Behinderungsparadigmen (kulturell-kognitiv), Organisationsformen und Lernorten (normativ), und bildungsbezogene Rechten (regulativ) - die den Wandel hin zur inklusiven Bildung bestimmen. 
Die globale Diffusion des Menschenrechts auf inklusive Bildung fordert daher etablierte Organisationsformen sonderpädagogischer Fördersysteme sowie kulturell verankerte Paradigmen von Bildung und Behinderung heraus. Durch die UN-BRK - eine wesentliche Errungenschaft der weltweiten Behindertenbewegung - ist dadurch auf globaler Ebene eine umfassende Reformagenda entstanden, die das gesamte institutionelle Bildungsgefüge in den Nationalstaaten betrifft.

Die UN-BRK ist darüber hinaus die erste UN-Konvention, welcher die Europäische Union beigetreten ist. Inklusive Bildungsziele finden sich entsprechend auch in der Disability Strategy der Europäischen Kommission - als eine von acht Prioritäten - wieder: „The specific goal is to promote inclusive education and lifelong learning for pupils and students with disabilities. [...] to ensure that people with disabilities receive the support required, within the general education system, to facilitate their education, and that effective individualised support measures are provided in environments that maximise academic and social development, consistent with the goal of full inclusion“" (European Commission 2014, S. 27-28).

Bezieht man die Entwicklung von Bildungssystemen auf die UN-BRK, dann steht als Voraussetzung der Zugang zum allgemeinen Bildungssystem und das Verbot des Ausschlusses von allgemeiner Bildung (no-rejection clause; United Nations 2013) im Fokus. Ziel ist also die Überwindung schulischer Exklusion, jedoch ohne Exklusion mit Segregation gleichzusetzen. Neben dem individuellen Recht auf Zugang zu allgemeinen Schulen unter Bereitstellung angemessener Vorkehrungen steht der systemische Wandel des Bildungssystems, welches Exklusion und Segregation überwinden muss. In diesem Zusammenhang stellt die 2013 veröffentlichte thematische Studie des UN-Hochkommissariats für Menschenrechte zum Recht auf Bildung von Menschen mit Behinderungen (United Nations 2013) heraus, dass die Implementierung des Rechts auf inklusive Bildung zweigleisig erfolgen muss: „firstly, nondiscrimination in mainstream schools for students with disabilities, reinforcing this right with reasonable accommodation; secondly, a systemic change that requires progressive realization and a transformation plan to counter exclusion and segregation" (United Nations 2013, S. 18). Auch das Handbuch für Parlamentarier zur Umsetzung der UN-BRK formuliert als Ziel dieses Prozesses das gemeinsame Lernen von behinderten und nichtbehinderten Kindern in derselben Schule (Deutscher Bundestag 2008, S. 83). Eine Beschulung in Sonderschulen auf Basis einer Behinderung kann folglich als Diskriminierung gewertet werden (Degener 2009; Mißling und Ückert 2014; Siehr und Wrase 2014).

Dem bereits 2007 erschienenen Bericht von Vernor Muñoz (A/HRC/4/29/Add.3), dem Sonderbeauftragten der Vereinten Nationen für das Recht auf Bildung, anlässlich seines Besuchs in Deutschland folgend, kann das segregierende Fördersystem als Barriere im Hinblick auf die Verwirklichung des Rechts auf inklusive Bildung kritisiert werden. Daher müssen Fördersysteme grundlegend verändert werden, wenn inklusive Schulbildung als Umsetzung des Rechts auf den Besuch einer wohnortnahen Schule ohne Auflagen (vgl. Latham \& Watkins 2009) Wirklichkeit werden soll. Für das Gelingen inklusiver, auf der UN-BRK basierender, Schulreformen bedarf es daher einer systematischen Herangehensweise um Reformhindernisse überwinden zu können, wie dies in der Bonner Erklärung zur inklusiven Bildung in Deutschland vom 20. März 2014 festgehalten ist (Deutsche UNESCO-Kommission 2014). 
Gleichwohl muss festgehalten werden, dass in den meisten Ländern weltweit die notwendigen Schritte der Weiterentwicklung - oder gar die Auflösung - von sonderpädagogischen Fördersystemen hin zur inklusiven Beschulung aller Kinder in einem Klassenraum noch nicht vollzogen wurde. Damit verbundene Herausforderungen sind weltweit und in nahezu allen Ländern ähnlich existent und beziehen sich auf die etablierten sonderpädagogischen Fördersysteme, denn welche Lernorte bereitgestellt werden, ist eng mit deren Auf-, Aus- oder Umbau verwoben. Notwendigerweise muss die Entwicklung inklusiver Schulsysteme deshalb vor dem Hintergrund institutionalisierter sonderpädagogischen Fördersysteme, welche für die Beschulung von als förderbedürftig wahrgenommenen Schülergruppen seit über zwei Jahrhunderten ausgebaut wurden, diskutiert werden (vgl. z. B. Richardson und Powell 2011). Die diesem Beitrag zugrundeliegende Definition inklusiver Bildung nimmt daher dezidiert auf den Lernort Bezug, wohlwissend, dass dieses räumliche Konzept noch wenig über die Qualität vielfältiger Bildungsangebote und Unterstützungsleistungen im Einzelfall auszusagen vermag: Segregation (Trennung des Unterrichts zwischen Gebäuden; Regelschulen und Sonder- bzw. Förderschulen), Separation (Trennung innerhalb eines Gebäudes; Sonderklassen in Regelschulen) und Inklusion (gemeinsamer Unterricht) (vgl. Powell 2009).

Um die Herausforderungen der UN-Behindertenrechtskonvention für Bildungssystementwicklung analysieren zu können werden in diesem Beitrag kontrastierende Systeme sonderpädagogischer Förderung und inklusiver Bildung untersucht und komparativ dargestellt. Die Auswahl von Deutschland, Island und Schweden für diesen Vergleich repräsentiert ein Spektrum innerhalb Europas in Bezug auf die Entwicklung inklusiver Schulbildung. Es bietet sich an Deutschland mit Schweden und Island - trotz unterschiedlicher demographischer, sozialer und politischer Kontextbedingungen - zu vergleichen, da im Fokus des Vergleichs das Verhältnis von offiziell klassifiziertem Förderbedarf, entsprechenden Lernortzuweisungen und Regularien als Indikatoren für die institutionelle Dimensionen sonderpädagogischer Förderung - steht. Dies geschieht vor dem Hintergrund der in der UN-BRK verankerten Prinzipien von Nicht-Diskriminierung und Teilhabe mittels angemessener Vorkehrungen.

Die zentrale Forschungsfrage lautet daher: Wie wird die Förderung von als förderbedürftig wahrgenommenen Schülerinnen und Schülern in diesen drei nationalen Kontexten gestaltet? Ist die offizielle Klassifizierung eines sonderpädagogischen Förderbedarfs (SPF) Voraussetzung für die Bereitstellung von Förderung? Notwendigerweise müssen hierbei nicht nur rechtliche, sondern auch kulturelle und normative Perspektiven berücksichtigt werden, die für die Entwicklung inklusiver Schulsysteme nicht nur in Deutschland hilfreich sind und zur aktuellen Diskussion um schulische Inklusion beitragen können. Denn mit Hilfe des länder- und institutionenvergleichenden Blicks kann auf die Kontingenz sonderpädagogischer Klassifikationssysteme und entsprechender Lernortzuweisungen unter sich veränderten rechtlichen Rahmenbedingungen - sowie deren Verschränkung - aufmerksam gemacht werden. Es geht bei diesem Vorhaben daher weniger um eine ,top down“ Wirkungsanalyse der UNBRK oder eine Sammlung von ,good practice“ oder „,best practice“ Beispielen als vielmehr um eine Analyse kontextspezifischer Entwicklungen in den drei institutionellen Dimensionen in Bezug auf schulische Inklusion. Dies folgt dem Anliegen sich mit der Komplexität der verwendeten Terminologien und etablierter Organisations- 
formen in den drei Ländern auseinanderzusetzen, um auf diese Weise die Zugänge zu inklusiver Bildung und die Folgen dieser Zugänge zu diskutieren (vgl. D’Alessio und Watkins 2009, S. 234). Ziel ist es so zum einen, Gründe für persistente Unterschiede der Fördersysteme zu analysieren und zum anderen mögliche Handlungsspielräume für inklusive Schulbildung zu ergründen.

In einem ersten Schritt wird die vergleichende Literatur zu sonderpädagogischen Fördersystemen dargestellt und die theoretische Basis des Vergleichs, welcher mittels neo-institutionalistischer Analyseansätze erfolgt, erörtert. In einem zweiten Schritt wird die historische Entwicklung der drei nationalen Schulsysteme mit Fokus auf die Beschulung von als förderbedürftig wahrgenommenen Schülerinnen und Schülern dargestellt. Abschließend werden auf der Basis des Vergleichs neue Perspektiven auf den deutschen Kontext eröffnet.

\section{Von anderen lernen - vergleichende und institutionelle Ansätze zur Erforschung sonderpädagogischer Fördersysteme und inklusiver Bildung}

Bereits von Anfang an war die Rezeption von Erfahrungen aus dem Ausland für die fachliche Entwicklung der Sonderpädagogik mitbestimmend (vgl. z. B. Klauer und Mitter 1987; Bürli 2006; Powell 2006; Biewer und Luciak 2010). Insbesondere vergleichende Ansätze sind hilfreich um die institutionellen, soziokulturellen und bildungspolitischen Kontextfaktoren herzuarbeiten, die zu unterschiedlichen Entwicklungsdynamiken der Fördersysteme geführt haben und die Möglichkeiten und Grenzen inklusiver Schulbildung setzen (vgl. Winzer und Mazurek 2011). Durch solche Vergleichsstudien wird es möglich, die sozialen und politischen Konstruktionen von „Befähigung“" und „Förderbedarf“, von „Behinderung“ und „Benachteiligung“ zu hinterfragen und Selbstverständlichkeiten in ihrer historischen Bedingtheit und Kontingenz zu erfassen (vgl. z. B. Powell 2011; Powell und Pfahl 2012). Derzeit gelten Konzepte aus den nordischen Ländern und dem angloamerikanischen Raum als Hauptquellen der Innovation (Biewer und Luciak 2010; Powell 2011). Unterschiede zwischen Ländern werden von Studien unterstrichen, die sich explizit deutsch-nordischen Vergleichen widmen (vgl. z. B. Kriwet 2006; Ratzki 2010; Schumann 2010; Barow und Persson 2011; Allemann-Ghionda 2013). Trotz der wachsenden Anzahl solcher internationalen Vergleiche (siehe z. B. auf deutsch Hans und Ginnold 2000; Albrecht et al. 2006; Bürli et al. 2009; Meijer 2010; Allemann-Ghionda 2013; Johnson 2013; Köpfer 2013) gibt es eine ganze Reihe von Forschungslücken, die sich von Ideen und Prinzipien, Interessen und Normen oder Standards bis hin zu Rechten und Ressourcen für Inklusion ziehen (siehe Powell 2011; Löser und Werning 2013).

Der hier verfolgte theoretische Ansatz nutzt den soziologischen Neo-Institutionalismus in der Erziehungswissenschaft (vgl. Meyer und Rowan 2006; Koch und Schemmann 2009) und wendet insbesondere Scotts (2008) institutionelles Säulenmodell auf die aktuelle Frage nach der Entwicklung inklusiver Schulsysteme im Ländervergleich an (vgl. Powell 2009). Institutionen bauen auf kognitiven, normativen und regulativen Strukturen und Verhaltensweisen auf. Institutionalisierung, verstanden als Entwicklungsprozess der Verfestigung sozialer Normen und Verhaltensmuster, kann somit nach Scotts (2008) klassischer Darstellung über drei Dimensionen 
oder Säulen analysiert werden, in diesem Fall: Behinderungsparadigmen und Bildungsideale als kulturell-kognitive Säule, die sonderpädagogische Profession und schulische Organisationsformen als normative Säule und die Bildungspolitik und Rechtsprechung als regulative Säule. Der Fokus dieses Beitrags liegt auf einer Analyse der Institutionalisierung von Klassifikationen und Kategorien des Förderbedarfs sowie diverser Organisationsformen - von Sonderschulen und Sonderklassen bis hin zur schulischen Integration und inklusiven Klassen - vor dem Hintergrund des in diesen drei Länderkontexten durch Unterzeichnung oder Ratifizierung der UN-BRK auf rechtlicher Ebene gegebenen Versprechens, inklusive Schulsysteme auf- und auszubauen.

\section{Daten und Fallauswahl}

Die Datenbasis des Vergleichs sonderpädagogischer Fördersysteme stützt sich zum einen auf den von der European Agency for Special Needs and Inclusive Education herausgegebenen Bericht „Special Needs Education Country Data“ (European Agency 2012) und auf nationale Bildungsberichte, zum anderen auf Gespräche mit Länderexperten und die Auswertung wissenschaftlicher Länderstudien. Aus der hier eingenommenen Makroperspektive können vor allem nationale Tendenzen dargestellt werden. Der Rückgriff auf die Daten der European Agency ist für diesen Vergleich von Vorteil, da diese Daten nach an Lernumwelten orientierten Kategorien systematisiert werden, die wiederum den in Kap. 1 vorgestellten Definitionen nahe kommen und folglich verbunden werden können: Sonderschulen, Sonderklassen oder inklusive Lernsettings. Von Segregation spricht die European Agency dann, wenn Schülerinnen und Schüler mit SPF in getrennten Gebäuden unterrichtet werden oder $80 \%$ des Tages oder mehr in Sonderklassen verbringen (European Agency 2012, S. 5). Dies wiederum reflektiert die Operationalisierung US-amerikanischer Daten auf Basis des Annual Report to Congress: nach Zeit in Regelklassen (vgl. Johnson 2013). Allerdings muss bei einer auf den Daten der European Agency basierenden Systematisierung von Förderquoten bzw. Klassifizierungsraten unbedingt beachtet werden, dass die sprachspezifischen Definitionen von Kategorien des Förderbedarfs in ihren vielfältigen kulturellen Bedeutungen zu verstehen sind. Deshalb wird hier lediglich die Tatsache der Notwendigkeit einer offiziellen Klassifizierung für die Ressourcenzuwendung als, zugegebenermaßen grober, Indikator genutzt. Das Ressourcen-Etikettierungs-Dilemma (Füssel und Kretschmann 1993) und die damit verbundene „Klassifizierungshemmschwelle“ (Powell 2011) wäre daher auf jeder Ebene, von der lokalen Schule bis zum nationalen System, zu bestimmen, kann hier jedoch nicht dargestellt werden.

Ein erster Überblick listet die auf der Basis der European Agency (2012) ermittelten Anteile der Schülerinnen und Schüler mit SPF an der Gesamtschülerschaft und in Sonderschulen oder -klassen auf. Darauf aufbauend werden diese Daten selbst als Ausdruck der Kontingenz von Klassifikationssystemen und entsprechender Lernortzuweisungen problematisiert. Im direkten Vergleich dieser Zahlen findet sich eine erstaunliche Varianz im Anteil der Schülerinnen und Schüler mit SPF an der Gesamtzahl aller Schülerinnen und Schüler (Förderquote). Auffallend ist zum ersten die große Bandbreite der Förderquoten. Hinsichtlich des offiziellen Anteils der Schüler 
Tab. 1 Förderquoten und -orte: Deutschland, Island und Schweden, 2010/2011.

(Quelle: Eigene Berechnungen auf Basis von Daten der European Agency (2012))

\begin{tabular}{llll}
\hline $\begin{array}{l}\text { Förderquote } \\
\text { (Anteil der Schülerinnen und Schüler mit SPF }\end{array}$ & Deutschland & Island & Schweden \\
$\begin{array}{l}\text { an allen Schülerinnen und Schüler) } \\
\begin{array}{l}\text { Segregierungs- und Separierungsquote (Anteil der Schülerin- } \\
\text { nen und Schüler mit SPF in Sonderschulen oder -klassen) }\end{array}\end{array}$ & $24,3 \%$ & $1,4 \%$ \\
\hline
\end{tabular}

mit SPF an der gesamten Schülerschaft bewegen sich Island $(24,3 \%)$ und Schweden $(1,4 \%)$ einmal am oberen und einmal am unteren Ende einer europäischen Skala der Förderquote, auf welcher Deutschland im Mittelfeld (5,5\%) liegt (siehe Tab. 1).

Während sich die Förderquote in Deutschland und Island auf alle Schülerinnen und Schüler bezieht, welche eine offizielle Bescheinigung hinsichtlich eines SPF haben, wird in Schweden gänzlich auf eine offizielle Definition SPF verzichtet und Schülerinnen und Schüler mit SPF in der allgemeinen Schule statistisch nicht erfasst (European Agency 2012; Paulsson et al. 2011). Während die European Agency daher von einer unbekannten Zahl von Schülerinnen und Schülern mit SPF spricht, welche in schwedischen Regelschulen lernen, gibt es an anderer Stelle Hinweise, dass für ca. $15 \%$ der schwedischen Schülerschaft sonderpädagogische Förderung in Regelschulen angeboten wird (vgl. D’Alessio und Watkins 2009, S. 242). Es bietet sich unserer Meinung nach allerdings nicht an, beide Zahlen zusammenzufassen und so eine Gesamtförderquote zu errechnen, da damit der schwedische Antiklassifizierungsansatz konterkariert würde. Entscheidend ist an dieser Stelle viel eher auf die Bedeutung und Relevanz von Klassifizierung als Differenzierungsprozess in Schulsystemen aufmerksam zu machen. Die statistische „Leerstelle“, welche im deutschen Fall in Bezug auf die verschiedensten Sonderklassen in den 16 deutschen Schulsystemen zu finden ist (siehe Blanck 2014), liefert vielmehr Hinweise auf die Logiken und Strukturen der Bildungssysteme und die in diesen zum Ausdruck kommende Relation von Klassifizierung und Lernort und schließlich dem Bildungsideal, wie später gezeigt wird. Daher ist diese Kontingenz in den Daten Ausdruck der Kontingenz von Terminologien; verschiedenen Verständnissen von SPF sowie deren offizieller und statistischer Erfassung.

Folglich steht die vergleichende Forschung im Themenbereich inklusiver Bildung vor großen Herausforderungen im Hinblick auf die Vergleichbarkeit der im Zusammenhang mit inklusiver Schulbildung verwendeten Terminologien, der Komplexität der gesetzlich regulierten Klassifikations prozesse von SPF als auch der Regelungen in Bezug auf die Lernorte (D'Alessio und Watkins 2009, S. 234). Um den damit verbundenen Logiken nachzuspüren werden daher im folgenden Kapitel die institutionellen Dimensionen vertiefend analysiert und die historische Entwicklung der Fördersysteme skizziert.

\section{Vergleich der Institutionalisierung sonderpädagogischer Fördersysteme und inklusiver Bildung in Deutschland, Island und Schweden}

In diesem Kapitel werden zunächst die drei Länder in Bezug auf die Institutionalisierung sonderpädagogischer Fördersysteme dargestellt. Dann werden, entlang der kulturell-kognitiven, normativen und regulativen Säulen der Institution sonderpäd- 
agogischer Förderung, entscheidende Charakteristika dieser drei Bildungssysteme verglichen. Die Vergleichsdimensionen lenken den Blick zuerst auf die mit den Fördersystemen verbundenen Begriffe, Kategorien und Klassifikationsysteme, welche die zugrundeliegenden Bildungsideale und Behinderungsparadigmen reflektieren (4.1). Danach wird dargestellt, welche Organisationsformen des Lernens und Förderns und damit Lernopportunitäten entstanden und gegenwärtig verfügbar sind (4.2). Zum Schluss wird Bezug genommen auf die regulative Dimension zu den Rechten und Bildungspolitik (4.3). Ziel ist es, so die Logiken der institutionalisierten Fördersysteme und der damit nationale Tendenzen pointiert darzustellen.

Deutschland: „Zunahme inklusiver Beschulung bei gleichzeitiger Beibehaltung schulischer Segregation und erhöhten Klassifizierungsraten“"

Beginnend um 1900 gab es in Deutschland eine lange Phase der Nachahmung, in welcher im gegliederten Bildungswesen Deutschlands die Hilfsschule (Sonderschule oder Förderschule) als eigenständige Schulform entwickelt wurde, welche der Logik „homogener" Lerngruppen folgt (Powell 2011). In der zweiten Phase, der Expansion und der Differenzierung seit etwa den 1950er-Jahren, kam es mit der universellen Schulpflicht und der sonderpädagogischen Förderung zu heterogeneren Schülerschaften, wobei die organisatorische Differenzierung nach Sonderschularten für die Förderung (,Sonderschulbedürftigkeit“) ausschlaggebend war. Trotz erfolgreicher Pilotprojekte des Gemeinsamen Unterrichts seit den 1970er Jahren (vgl. Schnell 2003; Preuss-Lausitz 2014) folgen elterliche, professionelle und politische Interessen hauptsächlich der ,institutionellen Logik“ des Bildungssystems, nämlich der Segregation. In dieser dritten - und bis heute andauernden Phase - zeichnet sich ein Konflikt zwischen der Persistenz der legitimierten Selektion in Sonderschulen (mit vielen Ressourcen, jedoch niedrigem Status) und dem Wandel hin zu inklusiver Bildung ab. Dabei steht der seit den 1960er Jahren stetige und bis heute andauernde Ausbau des Sonderschulwesens in Westdeutschland der Transformation des Bildungswesens in den neuen Bundesländern nach der Wiedervereinigung mit einer dramatischen Erhöhung der Sonderbeschulungsraten gegenüber (vgl. KMK 2012). Deshalb kann für Deutschland insgesamt nur bedingt von einer Entwicklung schulischer Integration oder Inklusion gesprochen werden, trotz vielfältiger lokaler und regionaler Erfolge des Gemeinsamen Unterrichts (vgl. z. B. Preuss-Lausitz 2001; Schöler et al. 2010; Moser 2012; Dorrance und Dannenbeck 2013), oft unter wissenschaftlicher Begleitung (vgl. zusammenfassend Preuss-Lausitz 2014).

Auch wenn sich Deutschland graduell in die Richtung inklusiver Bildung bewegt, vollzieht keines der 16 Bundesländer einen kompletten Pfadwechsel; wobei eine Divergenz unter den westdeutschen Flächenstaaten zu verzeichnen ist, mit Schleswig-Holstein als Vorreiter (weniger als $1 \%$ Sonderbeschulung im Primarbereich) und Bayern erhöht auf beinahe 5\% seit Ratifizierung der UN-BRK (Blanck et al. 2013; vgl. auch Dietze 2013). Derzeit kann in Deutschland in Hinblick auf die Implementierung der UN-BRK-Vorgaben insgesamt (noch) keine umfassende Transformation beobachtet werden. Obwohl der Inklusionsanteil bundesweit seit der UN-BRK-Ratifizierung 2008 von 18,4 auf 25\% gestiegen ist, bleibt wider Erwarten der Anteil der Schülerinnen und Schüler in Sonderschulen weitgehend stabil (4,6 zu 4,8\%), weil immer mehr Schülerinnen und Schüler einen sonderpädagogischen Förderbedarf 
attestiert bekommen (Anstieg von 6 auf 6,4\%) (Klemm 2013, S. 6ff.). ${ }^{2}$ Deshalb kann für die Bundesrepublik die graduelle Zunahme inklusiver Bildung bei gleichzeitiger Beibehaltung schulischer Segregation, einhergehend mit erhöhten Klassifizierungsraten bzw. Förderquoten festgestellt werden. In Deutschland fungiert die Klassifikation auf Basis eines an Behinderung gebundenen Begriffs von sonderpädagogischem Förderbedarf als Selektionskriterium und damit Mechanismus für die Zuweisung zu räumlich getrennten Lernorten. Daher ist die Sonderbeschulung für drei Viertel aller Schülerinnen und Schüler mit SPF weiterhin Realität.

Island: „Inklusion in einer Gesamtschule mit sehr hohen Klassifizierungsraten“ Im Jahr 1907 trat in Island ein Gesetz zur öffentlichen Bildung in Kraft. Die Formalisierung des Bildungswesens erfolgte jedoch erst einige Jahrzehnte später nach dem Zweiten Weltkrieg. Mit Gründung der Gesamtschule im Jahr 1974 versuchte man die Chancengleichheit für alle Schülerinnen und Schüler unabhängig ihrer sozialen Herkunft oder Fähigkeiten zu gewährleisten (für einen Überblick der Entwicklung der isländischen Schule, siehe Sigurðardóttir et al. 2014). In den 1970er Jahren wurde Egalität somit zum höchsten Prinzip der Bildungspolitik erhoben. Dieses Prinzip basiert auf zwei Säulen der derzeitigen Politik - Bildung für Alle (,Education for All“) und seit 1989 inklusive Bildung - spezifiziert und mittels eines rechtebasierten Fokus (Recht auf Bildung aller Kinder) weiter gestärkt (UNESCO 2008). Zuletzt wurde 2008 ein Rahmengesetz erlassen, welches die Bedeutung von öffentlichen Nachbarschaftsschulen für alle Kinder unterstreicht und damit unter dem Einfluss der Finanzkrise die Gesamtschule nicht abschwächt, sondern weiter zu stärken versucht (vgl. Sigurðardóttir et al. 2014).

In Island wird sonderpädagogische Förderung in unterschiedlicher Art und Weise angeboten, wobei die separierenden und segregierenden Organisationsformen quantitativ einen sehr geringen Anteil ausmachen. Es gibt (wenige) Sonderklassen, die halb- oder ganztags besucht werden, einige Sonderschulen oder Heimunterricht. Während sich der Anteil aller Schülerinnen und Schüler die klassifiziert werden nahezu auf ein Viertel ausgeweitet hat, ist die Zahl der Sonderschulen von sieben im Jahr 2002 auf drei im Jahr 2010 gesunken. Diese Zahlen verweisen auf die spezifischen Bedingungen eines Landes mit ungefähr 300.000 Einwohnern, wo eine Gesamtschule als pragmatische Lösung erachtet wird und einem starken Gemeinschaftsgefühl entspricht (vgl. Bjarnason 2003). ${ }^{3}$ Die meisten Schülerinnen und Schüler der sehr großen Gruppe der besonders Geförderten erhalten daher Unterstützung innerhalb der Regelklassen der wohnortnahen Schule. Hier wird Lernförderung in verschiedenen Fächern aber auch als persönliche Assistenz angeboten, z. T. individuell oder in Kleingruppen differenziert innerhalb der Regelklasse (European Agency 2014). Die vielfältigen Formen der Unterstützung müssen angepasst werden um den heterogenen Bedürfnissen gerecht zu werden (Egilson und Traustadottir 2009). Eine Überarbeitung des nationalen Curriculums 1996 fokussierte zudem mehr auf die individuellen Bedürfnisse der Schülerinnen und Schüler, mit dem Ziel diese zu stärken und unabhängiger zu machen (Arnesen und Lundahl 2006, S. 295). Insgesamt kann festgehalten werden, dass Island Inklusion in einer Gesamtschule ausbaut, dennoch an der offiziellen Klassifizierung festhält. In Island ist der gleiche Lernort rechtlich 
verankert, so dass die Klassifizierung nicht als Grundlage für Selektion und darauf basierender Segregation dient (wohl aber zu mehr Ressourcen führt).

Schweden: „Ein inklusives Gesamtschulsystem ohne Klassifizierung“

Bereits 1842 wurde die allgemeine Schulpflicht in Schweden eingeführt. Mit Blick auf schulische Inklusion blickt Schweden wie Island auf eine lange, nunmehr dreiBigjährige Tradition zurück, welche dem Prinzip einer „Schule für Alle“ folgt (European Agency 2012, S. 65). In den 1960er Jahren wurde eine große Bildungsreform eingeleitet, in deren Zuge Volksschulen in Gesamtschulen umgewidmet und somit die Trennung zwischen Volksschulen und speziellen Schulen weitgehend überwunden wurde. Seit den frühen 1970er Jahren ist ein Gesamtschulwesen flächendeckend realisiert. Neben den neunjährigen Gesamtschulen finden sich in Schweden wenige Spezialschulen (specialskolor) für Schülerinnen und Schüler mit Hör- oder Sehbeeinträchtigungen und Programme für Schülerinnen und Schüler mit intellektuellen Behinderungen (särskolor), die oft aufgrund der geringen Bevölkerungsdichte in die Grundschulen integriert sind (Barow und Persson 2011; Swedish Institute 2014). (Der Besuch von Gymnasien ist nach Abschluss der Gesamtschule vorgesehen.) In den 1980er Jahren wurde eine Perspektivenerweiterung vorgenommen, wonach im Lehrplan die Verantwortung der Schule für die Lernfortschritte aller Schülerinnen und Schüler hervorgehoben wurde und damit der Fokus für mögliche Faktoren bei Problemen weg vom Individuum hin zur Schule lenkt (Barow und Persson 2011, S. 23).

Die Bildungspolitik Schwedens ist jedoch seit Jahren starken Wandlungsprozessen unterworfen, vor allem im Zuge einer zunehmenden Privatisierung, die das ursprüngliche Konzept der Gesamtschule angreifen (Barow 2011; Barow und Persson 2011, S. 21; Schumann 2010). So schreibt das schwedische Schulgesetz von 2011 zwar weiterhin vor, dass alle Kinder den gleichen Zugang zu Bildung in den öffentlichen Schulen haben und diese selbst wählen können, jedoch verankert das Gesetz erstmals auch schultypenspezifische Lehrpläne und eine Wiedereinführung der Ausbildung zu „Speziallehrern“ (Barow und Persson 2011; Paulsson et al. 2011). Dies spricht zunächst nicht notwendigerweise gegen inklusive Bildung, tangiert aber das Bildungsideal einer ,Schule für Alle“. Trotz der starken Fundamente von Egalität, des Prinzips einer möglichst guten Schulbildung für Alle und des Rechts jeder Schülerin und jedes Schülers auf besondere Unterstützung (Allemann-Ghionda 2013, S. 137) muss festgestellt werden, dass aktuelle bildungspolitische Entwicklungen die Rahmenbedingungen für das breite Verständnis schulischer Inklusion in Frage stellen (vgl. Berhanu 2011). Dennoch wird in Schweden auf eine rechtliche Definition von SPF verzichtet und dies mit der Ablehnung von Klassifizierung und damit der Konstruktion von oft stigmatisierten Gruppen begründet. Unterstützung ist ein Recht der Schülerinnen und Schüler und steht allen zu, welche einen individuellen Bedarf haben - unabhängig der zugrundeliegenden Faktoren.

Nachdem die Institutionalisierung von sonderpädagogischem Förderbedarf und inklusiver Bildung skizziert wurde, sollen die drei Ländern nun anhand der drei institutionellen Dimensionen - kulturell-kognitiv, normativ und regulativ - tiefergehend verglichen werden. 
4.1 Behinderung im Bildungskontext: Begriffe, Kategorien und Klassifikationen der schulischen Behinderung (kulturell-kognitive Säule)

Die den verschiedenen Ländern zugrundeliegenden Definitionen der Zielgruppe zusätzlicher Fördermaßnahmen variieren erheblich. Als Basis einer gemeinsamen Dimensionalisierung verwenden wir die von der European Agency im Bericht „Special Needs Education Country Data 2012“ zusammengetragenen rechtlichen Definitionen SPF, welche schlagwortartig zusammengefasst werden (European Agency 2012, S. 26 f., 34, 65):

- Deutschland: spezifische Unterstützung für Schülerinnen und Schüler mit Behinderungen und Lernschwierigkeiten (je nach „Förderschwerpunkt“),

- Island: Unterstützung aufgrund von Behinderungen, emotionalen oder sozialen Schwierigkeiten,

- Schweden: keine rechtliche Definition von SPF.

Schweden verzichtet gänzlich auf eine rechtliche Definition von SPF und begründet dies mit der Ablehnung von Klassifizierung (Swedish Institute 2014). Das Recht auf flexible Unterstützung im Lernprozess steht im Mittelpunkt und lenkt daher den Fokus allgemein auf Lernschwierigkeiten, unabhängig der sie hervorrufenden oder bedingenden Faktoren, und erfasst somit statistisch nicht Schülerinnen und Schüler mit Unterstützungsbedarf in allgemeinen Schulen. Dieser Antiklassifizierungsansatz entspricht dem Leitbild einer ,Schule für Alle“ (European Agency 2012, S. 65), welche in Schweden für neun Jahre und in Island für zehn Jahre rechtlich verankert ist (Eurydice 2014). Aufgrund von Lern-, emotionalen oder sozialen Problemen, Behinderungen oder Krankheiten haben Schülerinnen und Schüler das Recht auf spezielle Unterstützung, welche auf einer Evaluation ihrer Bedürfnisse basiert.

In Island resultiert die Förderung innerhalb der wohnortnahen Vor-/Schule aus dem Recht aller Kinder und Jugendlichen auf Beratung und Betreuung durch Spezialisten. Wenn SozialpädagogInnen, BetreuerInnen oder MedizinierInnen Symptome einer Behinderung oder Beeinträchtigung eines Kindes vor Einschulung entdecken, informieren sie die Eltern, die dann eine der vier nationalen Agenturen (The State Diagnostic and Counselling Centre, Icelandic Organization of the Visually Impaired, The National Hearing and Speech Centre oder The Child and Adolescent Psychiatric Unit of the National Hospital) für weitere diagnostische Abklärung aufsuchen. Dies hat zur Folge, dass fast alle Kinder mit schwerwiegender Behinderung und sozialen oder emotionalen Schwierigkeiten bereits vor Schuleintritt, ihre Eltern und die betroffenen Vor-/Schulen der Gemeinde Unterstützung erhalten. Entsprechend des inklusiven Leitbilds isländischer Gesamtschulen haben alle Kinder ein Recht auf angemessenen Unterricht und spezialisierte Unterstützung. Förderung kann darüber hinaus bei Bedarf auch zu Hause, in einer medizinischen Einrichtung oder in einer Sonderklasse oder Sonderschule auf Wahl der Eltern erfolgen, wenn sie und die LehrerInnen meinen, das Kind könne keinen angemessenen Unterricht in der Gesamtschule erhalten (vgl. Eurydice 2014).

In einem stratifizierten Bildungswesen wie dem deutschen werden Schülerinnen und Schüler dagegen von Beginn ihrer Schulzeit an biologischen, kognitiven oder sozialen Standards gemessen, um festzustellen, ob sie von den durchschnittlich zu erwarten- 
den Fähigkeiten, Leistungen oder Verhaltensweisen abweichen. Dem zugrunde liegt „die Fiktion von homogenen Lerngruppen“ (Werning 2010, S. 285; Preuss-Lausitz 2011). Trotz der seit 1994 in Deutschland geltenden „Förderschwerpunkte“ entspricht die Klassifizierungslogik immer noch den Strukturen des Sonderschulsystems. Dabei sollen sich bei den sieben Förderschwerpunkten die an Behinderung orientierten Definitionen nun an pädagogischen Erfordernissen orientieren, rekurrieren aber weiter auf Behinderung. Auch im Bereich inklusiver Bildung wird der Fokus auf Kinder und Jugendliche mit Behinderungen gelegt, wie dies der Beschluss der KMK vom 20.10.2011 bereits im Titel trägt und folglich die Ausweitung inklusiver Bildungsangebote als einen „Schwerpunkt sonderpädagogischen Handelns“ sieht (KMK 2011, S. 2). Klassifizierung fungiert dabei als Auslöser für eine Zuweisung zu Sonderschulen. Die Kategorie von Lernbehinderung gilt in Deutschland mit einem Anteil von über 40\% als der größte Förderschwerpunkt (Klemm 2013, S. 12), wobei die Dominanz dieser Kategorie in den letzten Jahren zurückgegangen ist (Autorengruppe Bildungsberichterstattung 2014, S. 163). Hier spiegelt sich jedoch wider, dass soziale Ungleichheiten durch die Sonderschule oft reproduziert, soziale Probleme in individualisierende Kategorien von Lernbehinderung umgedeutet und legitimiert werden (Pfahl 2011) und dies der Befähigung der Schülerinnen und Schüler entgegengewirkt (Pfahl 2012). Festzuhalten bleibt daher, dass in den meisten Länderkontexten die Gruppen beeinträchtigter, benachteiligter und „schulisch behinderter“ Kinder und Jugendlicher große Überlappungen aufweisen.

Diese unterschiedlichen Zugänge in der rechtlichen Definition eines SPF müssen in Verbindung gebracht werden mit den vorherrschenden Bildungsidealen und Behinderungsparadigmen. Während Leistungs- und Begabungsorientierung im deutschen Kontext zentral ist (Pfahl 2011, 2012), ist dies Egalität in Island (Sigurðardóttir et al. 2014) und Schweden (Allemann-Ghionda 2013). In Island gehört neben Toleranz auch die Partizipation an einer demokratischen Gesellschaft zu den idealisierten Werten; Gemeinschaft und Teilhabe sind auch in Schweden stark im Fokus (vgl. Blossing et al. 2014). Das an Leistung orientierte Schulwesen und nach Behinderungsarten ausdifferenzierte Sonderschulwesen Deutschlands wirkt auf ein Verständnis von Behinderung vor allem als ein individuelles Defizit oder als individuelle Abweichung von der Norm (Powell 2011). Dahingegen wird in den beiden nordischen Ländern Behinderung stärker als Aspekt der menschlichen Vielfalt gewertet, wobei das Modell der Gesamtschule Werte wie Egalität, Progressivität und Pragmatismus ins Zentrum stellt (Antikainen 2006, S. 240; Barow und Persson 2011; European Agengy 2014; Swedish Institute 2014). Idealtypisch können im Vergleich der nordischen Fälle Gemeinsamkeiten mit Blick auf das Bildungsideal und vorherrschende Leitbild und ähnliche gesellschafts- statt individuenzentrierte Behinderungsparadigmen festgestellt werden. Dieser Kontrast wird im unterschiedlichen Zugang der Klassifizierungsansätze weiter verdeutlicht (siehe Tab. 2). ${ }^{4}$ Auch wenn alle drei Länder eine eugenische Tradition im 20. Jahrhundert (inkl. der Zwangssterilisierung, vgl. Weindling 1999) zu überwinden haben, symbolisiert die Unterzeichnung der UN-BRK die Sensibilisierung gegenüber dieser Diskriminierung. 
Tab. 2 Kulturell-kognitive Säule: Bildungs- und Behinderungsparadigmen. (Quelle: Eigene Darstellung auf Basis von Allemann-Ghionda 2013; Antikainen 2006; Barow und Persson 2011; Powell 2011; Sigurðardóttir et al. 2014; Swedish Institute 2014)

\begin{tabular}{llcc}
\hline & Deutschland & Island & Schweden \\
\hline $\begin{array}{l}\text { Ideal im } \\
\text { Bildungssystem }\end{array}$ & Leistung & Egalität \\
$\begin{array}{l}\text { Leitbild } \\
\begin{array}{l}\text { Gesellschaftliches Be- } \\
\text { hinderungsparadigma } \\
\text { (Idealtyp) }\end{array}\end{array}$ & $\begin{array}{l}\text { Homogene Lerngruppen } \\
\text { Behinderung als indivi- } \\
\text { duelles Defizit }\end{array}$ & $\begin{array}{c}\text { Heterogene Lerngruppen („Eine Schule für Alle“) } \\
\text { Behinderung als Aspekt der Vielfalt bzw. als Be- } \\
\text { ziehung zwischen Individuum und Umwelt }\end{array}$ \\
\hline
\end{tabular}

\subsection{Lernopportunitäten, Fördersysteme und inklusive Bildung (normative Säule)}

Behinderungsbegriffe und deren Einfluss auf die Organisationsformen der sonderpädagogischen Unterstützung sind von zentraler Bedeutung für Lernopportunitäten. Im Folgenden werden daher die in Tab. 1 präsentierten Tendenzen als Ausdruck von Bildungs- und Behinderungsidealen nun tiefergreifend vergleichend dargestellt.

Die Struktur des deutschen Bildungssystems ist mehrfach gegliedert: auf die vierjährige (sechsjährige) gemeinsame Grundschule folgt eine Reihe von Sekundarschulen (Gymnasien; Gesamtschulen; Sekundar-, Mittel- oder Realschulen; Hauptschulen; Sonderschulen). Die bedeutsamste Unterscheidung ist die zwischen Regel- und Sonderschulen, da sie eine binäre Struktur verankert und die Förderung noch stark an die Sonderschulen bindet, auch wenn zunehmend ambulante Förderdienste existieren (Blanck et al. 2013). Während in Deutschland ein hoch differenziertes Sonderschulwesen etabliert ist, stellt die Partizipation einzelner Kinder im Einheitsschulsystem in Island das primäre Ziel dar. Grundlegend ist ein rechtebasiertes Verständnis von Bildung, welches sich nicht an diagnostischen Kategorien orientiert, sondern auf bildungsspezifischen Bedürfnissen beruht - und weitergehend versucht die Barrieren, welche diese verursachen, zu reduzieren (European Agency 2014). In Island ist eine inklusive zehnjährige gemeinsame Pflichtschule für Alle vorgesehen, wohingegen das auf Wahlmöglichkeiten hin orientierte Schweden eine neunjährige gemeinsame Grundschule bereitstellt, Gymnasien nach Abschluss dieser, Spezialschulen und Sonderschulen, wobei private Angebote zunehmen (Barow und Persson 2011; Swedish Institute 2014). Diese Wahlmöglichkeiten sind Ausdruck einer Systemstruktur entlang eines Kontinuums von möglichen Förderorten. In Schweden werden die meisten (96\%) der relativ wenigen Schülerinnen und Schüler mit einem offiziell festgestellten und statistisch erfassten SPF in Sonderklassen unterrichtet. Obwohl die Nutzung medizinischer Kategorien für bildungsbezogene Zwecke abgelehnt wird, so dass Unterstützung in der allgemeinen Schule nicht an eine offizielle Klassifizierung gebunden ist, ist der Bezug auf Behinderungen für Separation in Sonderklassen weiterhin gegeben. Dies hat zur Folge, dass der statistisch erfasste häufigste Lernort für offiziell mit einem SPF klassifizierte Schülerinnen und Schüler in Deutschland die Sonderschule (Förderschule) ist, während dies in Schweden Sonderklassen sind (weil die überwiegende Mehrheit der Unterstützungsleistungen ohne offizielle Klassifizierung bereitgestellt werden) und in Island inklusive Klassen (European Agency 2011, 2012) (Tab. 3). 
Tab. 3 Normative Säule: Organisationsformen. (Quelle: Eigene Darstellung auf Basis von European Agency 2012, 2014; Eurydice 2014)

\begin{tabular}{|c|c|c|c|}
\hline & Deutschland & Island & Schweden \\
\hline $\begin{array}{l}\text { Statistisch häufigster } \\
\text { Lernort für offiziell } \\
\text { klassifizierte Schülerin- } \\
\text { nen und Schüler }\end{array}$ & Sonderschulen & Regelklassen & Sonderklassen \\
\hline Organisationsformen & $\begin{array}{l}\text { Vierjährige gemeinsame } \\
\text { Grundschule, Regel- } \\
\text { schulen, Gymnasien, } \\
\text { Förderschulen }\end{array}$ & $\begin{array}{l}\text { Zehnjährige gemein- } \\
\text { same Pflichtschule, } \\
\text { Sonderschulen }\end{array}$ & $\begin{array}{l}\text { Neunjährige gemein- } \\
\text { same Grundschule, } \\
\text { Gymnasien (Sekundar- } \\
\text { stufe II), Sonderschu- } \\
\text { len, Spezialschulen }\end{array}$ \\
\hline Förderung & $\begin{array}{l}\text { Gebunden an den Ort } \\
\text { und damit die Struktur } \\
\text { des Schulsystems }\end{array}$ & $\begin{array}{l}\text { In der allgemeinen } \\
\text { Schule (reguläre } \\
\text { Klasse); in Ausnahme- } \\
\text { fällen in Sonderklassen } \\
\text { oder Sonderschulen (auf } \\
\text { Antrag) }\end{array}$ & $\begin{array}{l}\text { Vielfältige Fördermög- } \\
\text { lichkeiten hinsicht- } \\
\text { lich des Ortes, der } \\
\text { Gestaltung und der } \\
\text { Unterstützung }\end{array}$ \\
\hline
\end{tabular}

\subsection{Einfluss der UN-BRK und Ausweitung der inklusiven Bildung (regulative Säule)}

Alle drei Länder haben die UN-BRK im Jahr 2007 unterzeichnet. Deutschland und Schweden haben die Konvention und das Zusatzprotokoll im Jahr 2009 und 2008 ratifiziert, wohingegen Island diesen zweiten Schritt noch nicht gegangen ist. Schweden hat dem Ausschuss für die Rechte von Menschen mit Behinderungen (Committee on the Rights of Persons with Disabilities), einem unabhängigen Expertengremium, welches die Implementierung der UN-BRK überprüft (OHCHR 2014), im Jahr 2011 den ersten Staatenbericht zur Überprüfung vorgelegt (CRPD/C/SWE/1), welcher im April 2014 begutachtet wurde. In den sogenannten Concluding Observations $(\mathrm{CPRD} / \mathrm{C} /$ SWE/CO/1) lobt der Ausschuss gleich zu Beginn Schwedens inklusives Bildungssystem, in welchem nur 1,5\% der Schülerinnen und Schüler, in Übereinstimmung mit der familiären Zustimmung, außerhalb des regulären Klassenzimmers unterrichtet werden. Weiterhin würdigt der Ausschuss neue rechtliche Regelungen, nach welchen die Verweigerung angemessener Vorkehrungen (reasonable accommodation) als Diskriminierung gilt. Nichtsdestotrotz zeigt sich der Ausschuss mit Bezug auf Artikel 24 besorgt über Berichte, dass Schulen den Zugang für bestimmte Schülerinnen und Schüler mit Behinderungen auf Basis organisatorischer oder wirtschaftlicher Schwierigkeiten verweigern können oder einige Kinder, welche umfassende Unterstützung benötigen, Schulen aufgrund eines Mangels dieser nicht besuchen können.

In Deutschland gibt es einen Nationalen Aktionsplan zur Umsetzung der UN-BRK (BMAS 2011), einen ersten Staatenbericht (CRPD/C/DEU/1) und einen umfassenden Parallelbericht zu diesem, welcher von der BRK-Allianz (2013), einer Vereinigung zivilgesellschaftlicher Gruppen, verfasst wurde. Die Beratung und Prüfung ist für April 2015 vorgesehen und kann gespannt erwartet werden. Denn im Mai 2014 hat der Ausschuss eine Fragenliste zur Berichtsprüfung verfasst (CRPD/C/DEU/Q/1), in welcher explizit nach den Maßnahmen der Bundesregierung gefragt wird, welche die Erfüllung der Vorgaben des Artikels 24 UN-BRK durch die Länder sicherzustellen versuchen. Weiter wird nach detaillierten Angaben zu den Plänen der Länder und 
deren Verständnis aus der UN-BRK resultierender rechtlichen Verpflichtungen im Bereich Bildung gefragt.

In Island hat das Icelandic Human Rights Centre die Regierung ermahnt, die UNBRK zu ratifizieren. Das Wohlfahrtsministerium bereitet derzeit eine umfassende Klärung der Situation von Menschen mit Behinderung und Behindertenpolitik vor, die 2014 abgeschlossen werden soll (Icelandic Human Rights Centre 2014).

Mit Bezug auf die regulative Säule und damit den gesetzlichen Regelungen sonderpädagogischer Förderung, welche den Prinzipien der UN-BRK entsprechen, kann an dieser Stelle daher (noch) keine ausführliche Erörterung erfolgen. Die UN-BRK wird zwar intensiv diskutiert, diese Diskussionen schlagen sich allerdings noch nicht in vollem Umfang in entsprechenden Gesetzen nieder. Eine vom Deutschen Institut für Menschenrechte in Auftrag gegebene Studie stellt daher für Deutschland fest, dass sich die Landesschulgesetze seit Ratifizierung der UN-BRK an die dort veränderten menschenrechtlichen Vorgaben angepasst und geändert haben, jedoch ,,(k)ein Land... alle im Recht auf inklusive Bildung angelegten Kriterien (erfüllt)“ (Mißling und Ückert 2014, S. 7; vgl. weiterführend Blanck 2014). Die besondere Herausforderung besteht darin, dass das Teilhaberecht, das innerhalb von Schulen eine Fortsetzung staatlicher Leistungen voraussetzt, und das Diskriminierungsverbot, welches die Unterlassung sonderpädagogischer Förderung in der Regelschule verbietet, konvergieren (vgl. Siehr und Wrase 2014, S. 182).

Um die in der UN-BRK angelegten Prinzipien regulativ zu verankern, müssen daher explizit Entscheidungen getroffen werden im Hinblick auf Klassifikationssysteme und Klassifizierung sowie Lernortzuweisungen. ${ }^{5}$

\subsection{Zum Verhältnis von Klassifizierung und Lernort - synthetischer Vergleich institutioneller Dimensionen}

Die vergleichende Gegenüberstellung entlang der diskutierten institutionellen Säulen legt abschließend den Fokus auf das Verhältnis von Begriffen, Kategorien und Klassifizierungen des SPF und darauf aufbauenden Lernortzuweisungen in unterschiedlichen Organisationsformen des Schulsystems. Die Analyse zeigt verschiedene Zugänge: Klassifizierung ohne Segregation (Island), Antiklassifizierungsansatz, aber Separation einer kleinen Minderheit (Schweden) und Klassifizierung als Auslöser für Segregation der Mehrheit der klassifizierten Kinder (Deutschland). Während in Deutschland der Trend zu Segregation mit der Sonderschule als häufigstem Lernort für Schülerinnen und Schüler mit SPF ungebrochen ist, nähert sich Island einem inklusiven System an, in welchem fast alle Kinder gemeinsam lernen und das, obwohl sehr viele Schülerinnen und Schüler offiziell als förderbedürftig wahrgenommen und klassifiziert werden. Hingegen werden im Gesamtschulsystem Schwedens nur sehr wenige Kinder klassifiziert, da Ressourcen ohne offizielle Diagnosen bereitgestellt werden können. Die meisten der im Vergleich sehr viel kleineren Gruppe von Schülerinnen und Schülern mit offiziellem SPF werden jedoch in Sonderklassen unterrichtet. Der statistisch erfasste Anteil der Schülerinnen und Schüler in Sonderschulen an allen Schülern (Segregationsquote) zeigt daher folgende Disparitäten: während Deutschland mit 78,7\% eine der höchsten Sonderbeschulungsquoten in Europa aufweist, gehören Island (1,3\%) und Schweden (4\%) zu den Ländern, neben Norwegen 
und Italien, die Sonderschulen als segregierende Organisationsformen stark reduziert haben (European Agency 2012). Bei diesen Zahlen ist erneut zu beachten, dass ihre Erhebung nicht auf den gleichen Regeln und Verfahren beruht und sie damit Ausdruck der landesspezifischen Logiken selbst sind. Diese Analyse unterstreicht daher die Zusammenhänge der Inklusions- und Segregationsquoten mit den kulturell-kognitiven, normativen und rechtlichen Grundlagen der jeweiligen Schulsysteme. In Island und Schweden bildet die auf dem Prinzip der Egalität aufbauende Gesamtschule die normative Basis des Schulsystems, welche sich auf regulativer Ebene im Recht auf Bildung, persönliche Entwicklung und angemessene Unterstützung im prioritär vergesehenen gemeinsamen Unterricht widerspiegelt. Dahingegen verfügt das stratifizierte und an Leistung orientierte deutsche Schulsystem über ein differenziertes Angebot an Schulformen sowie ein ausgebautes Sonderschulwesen. In einem solchen führt Klassifizierung in den meisten Fällen zu Segregation, wohingegen ein solches Entweder/Oder in den nordischen Gesamtschulsystemen größtenteils ausbleibt.

Im Vergleich der Klassifikationssysteme und damit verbundener Lernopportunitäten wird deutlich, dass an Sonderschulen orientierte Bildungssysteme einem flexiblen Bereitstellen von Unterstützung und einem an Bildungsmöglichkeiten orientierten Verständnis von Inklusion entgegenstehen können. Grund hierfür ist, dass das Klassifizieren von Schülern und Schülerinnen den Mechanismus darstellt, an welchen die Vergabe von Ressourcen und die Gewährung von Rechten in Bildungseinrichtungen geknüpft ist. Dadurch können Prozesse des Klassifizierens dazu führen, dass Einzelne als „defizitär“" oder ,abweichend“ wahrgenommen werden, ohne die positiven Aspekte der individualisierten Lernförderung optimal auszuschöpfen (vgl. Kottmann 2006). Der Prozess der Klassifizierung zieht so nicht nur Ressourcen, sondern meist auch Stigmatisierung und räumliche Trennung nach sich, die die Befähigung der Individuen vermindern können (vgl. Pfahl 2011, 2012).

Es macht daher einen Unterschied, ob die Zugehörigkeit eines jeden Kindes zur allgemeinen Schule und Klasse ohne Auflagen rechtlich verankert ist oder ob Schülerinnen und Schüler erst durch Erarbeitung einer Anpassungsleistung an vorherrschende Normalitätsvorstellungen den Zugang zur allgemeinen Schule und Klasse erhalten. Anstatt Voraussetzungen für die Inklusion in eine Regelklasse vorzusehen, wird in Deutschland eine Begründung für Ressourcenzuwendung verlangt. Dies geht einher mit einem Wechsel der Perspektive im Hinblick auf den Akteur, welcher eine Anpassungsleitung vollbringen muss - das Individuum, um in der Regelklasse als nicht störend, im besten Sinne angepasst, wahrgenommen zu werden, oder die Umwelt im Sinne der Schule und LehrerInnen. Die bildungsbezogene Entwicklung wird somit zum Ankerpunkt der Förderung, die eine Identifizierung von Bedarfen und die entsprechende Anpassung der Lernumgebung zur Folge hat und nicht automatisch die Zuweisung zu einem bestimmten Lernort vorsieht. An der Schnittstelle Klassifikation/ Lernort kann die Mitgliedschaft in Förderbedarfskategorien gänzlich unterschiedliche Folgen für die individuelle Schullaufbahn haben in Abhängigkeit der Bildungssystemstrukturen wie auch der Rechtsprechung bezüglich inklusiver Bildung.

Zusammenfassend kann konstatiert werden, dass sich erhebliche Unterschiede bei den „Schwellen“ der Klassifizierung als „förderbedürftig“ und der Einschulung im sonderpädagogischen Bereich feststellen lassen. Der entscheidende Unterschied 
scheint zu sein, ob zusätzlicher Förderbedarf als Anspruch und Recht oder als Makel verstanden wird und somit entweder zu Unterstützung in der Regelklasse oder zur Aussonderung führt. Erfolgversprechend scheinen Ansätze, die eine Separierung oder gar Segregierung nur mit Begründung und im Ausnahmefall vorsehen und gleichzeitig den nötigen Freiraum in der Förderung eröffnen, sowohl im Hinblick auf Ressourcen als auch Orte, Dauer, Arrangements und Organisation. Eine Flexibilisierung der Lernumgebung ist daher unerlässlich um den Vorgaben der UN-BRK im Hinblick auf Teilhabe gerecht werden zu können.

\section{Schlussfolgerungen}

In vergleichenden Analysen werden sowohl die globale Herausforderung inklusiver Bildung deutlich als auch die unterschiedlichen Ausgangslagen nationaler und regionaler Bildungssysteme. Wie in anderen Ländervergleichen konnte hier die institutionelle Entwicklung nur skizzenhaft dargestellt werden. Dies ist allerdings eine notwendige Voraussetzung für die weiterführende Untersuchung inklusiver, auf der UN-BRK basierender Bildungspolitiken, welche das Menschenrecht auf inklusive Bildung ernstnehmen. Weitere historische und vergleichende Perspektiven und Studien sind in diesem Zusammenhang unabdingbar.

Unsere Analyse zeigt, dass Bildungsideale, Kategorien des SPF und Schulstrukturen entscheidenden Einfluss auf die Umsetzung des Rechts auf inklusive Bildung haben und nehmen. Eine Überwindung der prinzipiellen Exklusion (also kein Zugang zu formaler Bildung), wie sie in den 1960er-Jahren in Deutschland erreicht wurde, kann auf Basis dieses Vergleichs nur als der erste Schritt auf dem Weg zur größtmöglichen Teilhabe an formal organisierten Lernmöglichkeiten gewertet werden. Die gegenwärtige Beibehaltung von Förderschulen bei gleichzeitiger Zunahme von schulischer Inklusion in Deutschland - zumal erst seit 2001 statistisch erfasst und in den Bundesländern seither unterschiedlich ausgeweitet (vgl. Autorengruppe Bildungsberichterstattung 2014, S. 179) - bestätigt die Dualität der Strukturen. Im deutschen System kann sich Inklusion aktuell also vornehmlich auf sozialer Ebene und nur für die Schülerinnen und Schüler vollziehen, welche an allgemeinen Schulen lernen. Denn ob „Sonderschulbedürftigkeit“ (1950er-Jahre bis 1994) oder die aktuellen „Förderschwerpunkte“ (seit 1994): Das deutsche Schulsystem überkommt bisher nicht seine Orientierung an Behinderung und der Förderschule als Hauptorganisationsform für klassifizierte Schülerinnen und Schüler. Dies steht der aktuellen bildungspolitischen Zielsetzung der Inklusion und damit der Überwindung der Segregation und letztlich dem Lernen aller Schülerinnen und Schüler in gemeinsamen Klassen entgegen (vgl. Powell 2011; Klemm 2013).

Hingegen ist für die Bildungspolitiken Islands und Schwedens charakteristisch (geworden), dass sie im Großen und Ganzen auf soziale Inklusion abzielen und dem grundlegenden Prinzip bildungsbezogener Chancengleichheit folgen (Arnesen und Lundahl 2006, S. 291, 296). Jedoch bestehen wichtige Differenzen in der Art und Weise, wie Förderbedarfe erfasst und Kinder und Jugendliche in Schulen bezeichnet werden. Daher wird in Island und Schweden Inklusion auf sozialer, räumlicher und 
administrativer Ebene erreicht, darüber hinaus in Schweden zudem auf terminologischer Ebene verankert (Antiklassifizierungsansatz).

Dilemmata der demokratischen Schule und Fragen der kompensatorischen Wirkung der Sonderpädagogik bleiben fundamentale Debatten und große Herausforderungen in allen drei Ländern. Während in Deutschland die Diskussion um Inklusion ohne empirische Fokussierung der schulischen Inklusion geführt wird und somit stark ideologisch geführte Debatten überwiegen, fokussiert diese in Schweden auf eine wissenschaftliche Auseinandersetzung mit längst ausgebauten inklusiven Schulen (vgl. Kriwet 2006); in beiden Ländern bleiben die hohen Segregationsraten (Deutschland) und niedrige Separationsraten (Schweden) stabil, trotz Ratifizierung der UN-BRK. Im Vergleich der beiden nordischen Länder können die Herausforderungen im deutschen Kontext zur Verwirklichung inklusiver Schulbildung auf die „strukturelle Selektivität des Schulsystems, die Diskussion über outcome-Steuerung und Standards sowie das Fehlen eines umfassenden Gesamtschulsystems" (Werning 2010, S. 285) zurückgeführt werden. Jedoch zeigt der Vergleich, dass die institutionelle Transformation im Zuge der Ratifizierung in allen drei Säulen erfolgen und die Wechselverhältnisse berücksichtigt werden müssen. Dieser Beitrag hat durch die ländervergleichende Perspektive und die analytische Unterscheidung der Säulen das kontextspezifische Verhältnis von Klassifizierungssystemen und Lernorten herausgearbeitet. Die beiden nordischen Länder kommen mit ihrer Orientierung an Egalität und den etablierten Gesamtschulsystemen den in der UN-BRK verankerten Prinzipien aktuell bereits näher als Deutschland. Für alle Länder bleibt die Realisierung eines umfassenden Menschenrechts auf inklusive Bildung eine Herausforderung.

\section{Anmerkungen}

1 Während der 6. Session des Ad-Hoc-Committees, welches die Entwürfe zur UN-BRK beraten hat, zogen sich die Beratung des auf Bildung bezogenen Artikels über drei Tage hinweg (2.8.-4.8.2005), so lange wie zu keinem anderen Bereich (United Nations 2014b).

2 Nach Zahlen des Nationalen Bildungsberichts 2014 hat sich die Förderquote insgesamt von 2000/01 bis 2012/13 von 5,3 auf 6,6 \% erhöht - dies entspricht einer halben Million Kinder und Jugendlicher-, wobei die größten Zunahmen in den Förderschwerpunkten Sprache (5 auf $11 \%$ ) und emotionale und soziale Entwicklung (7 auf 14\%) der Reduzierung um ein Viertel im größten Förderschwerpunkt, nämlich Lernen (54 auf 40\%), gegenüberstand (Autorengruppe Bildungsberichterstattung 2014, S. 163).

3 Wobei andere kleinere Länder wie beispielsweise Luxemburg eine deutlich höhere Segregationsquote aufweisen (im Jahr 2011/12 verblieben lediglich 38\% aller Grundschüler mit Förderbedarf im Regelschulbereich der Grundschule) (Limbach-Reich und Powell im Druck).

4 Aus Platzgründen kann hier nicht auf intranationale Unterschiede eingegangen werden, etwa der sozialen und politischen Disparitäten innerhalb von Ländern und ihren Bildungssystemen (siehe z. B. Blanck et al. 2013 zur unterschiedlichen Umsetzung der UN-BRK in Bayern und Schleswig-Holstein). Hier stellen wir nationale Tendenzen dar, welche auf Grundlage der aktuellen Datenbasen möglich sind.

5 Aus diesem Grund wäre es ferner zu begrüßen, wenn der Ausschuss für die Rechte von Menschen mit Behinderungen neben den bereits veröffentlichten Allgemeinen Kommentaren (general comments) zu Artikel 9 (Zugänglichkeit) und Artikel 12 (Gleiche Anerkennung vor dem Recht) einen weiteren zum Recht auf Bildung erarbeiten würde (OHCHR 2014) um dieser Offenheit begegnen zu können. 


\section{Literatur}

Albrecht, F., Bürli, A., \& Erdélyi, A. (Hrsg.). (2006). Aspekte internationaler Heil- und Sonderpädagogik. Bad Heilbrunn: Klinkhardt.

Allemann-Ghionda, C. (2013). Bildung für Alle, Diversität und Inklusion. Paderborn: Schöningh.

Antikainen, A. (2006). In Search of the Nordic Model in Education. Scandinavian Journal of Educational Research, 50(3), 229-243.

Arnesen, A., \& Lundahl, L. (2006). Still Social and Democratic? Inclusive Education Policies in the Nordic Welfare States. Scandinavian Journal of Educational Research, 50(3), 285-300.

Autorengruppe Bildungsberichterstattung (2014). Bildung in Deutschland 2014. Ein indikatorengestützter Bericht mit einer Analyse zur Bildung von Menschen mit Behinderungen. Bielefeld: Bertelsmann Verlag.

Barow, T. (2011). Vorbild oder Zerrbild? Außen- und Innenperspektive auf inklusive Bildung in Schweden. Zeitschrift für Inklusion, 2011(4). http://www.inklusion-online.net/index.php/inklusion-online/ article/view/78/78. Zugegriffen: 08. April 2014.

Barow, T., \& Persson, B. (2011). Die Sonderpädagogik in der bildungspolitischen Debatte Schwedens. Sonderpädagogische Förderung heute, 56(1), 20-32.

Berhanu, G. (2011). Challenges and Responses to Inclusive Education in Sweden. In A. J. Artiles, E. B. Kozleski, \& F. R. Waitoller (Hrsg.), Inclusive Education Examining Equity on Five Continents (S. 101-119). Cambridge: Harvard Education Press.

Biewer, G., \& Luciak, M. (2010). Der internationale Vergleich in der Sonderpädagogik. Enzyklopädie Erziehungswissenschaft Online. doi:10.3262/EEO05100093

Bjarnason, D.S. (2003). School Inclusion in Iceland: The Cloak of Invisibility. New York: Nova Science.

Blanck, J.M. (2014). Organisationsformen schulischer Integration und Inklusion. Eine vergleichende Betrachtung der 16 Bundesländer. WZB Discussion Paper.

Blanck, J.M., Edelstein, B., \& Powell, J.J.W. (2013). Persistente schulische Segregation oder Wandel zur inklusiven Bildung? Die UN-Behindertenrechtskonvention und Reformmechanismen in den deutschen Bundesländern. Schweizerische Zeitschrift für Soziologie, 39(2), 267-292.

Blossing, U., Imsen, G., \& Moos, L. (Hrsg.) (2014). The Nordic Education Model: ,A School for All Encounters Neo-Liberal Policy. Heidelberg: Springer.

BMAS (2011) = Bundesministerium für Arbeit und Soziales. (2011). Unser Weg in eine inklusive Gesellschaft. Der Nationale Aktionsplan der Bundesregierung zur Umsetzung der UN-Behindertenrechtskonvention. Berlin: Bundesministerium für Arbeit und Soziales.

BRK-Allianz (2013) = Behindertenrechtskonvention-Allianz. (Hrsg.) (2013). Für Selbstbestimmung, gleiche Rechte, Barrierefreiheit, Inklusion! Erster Parallelbericht der Zivilgesellschaft zur Umsetzung der UN-Behindertenrechtskonvention. Berlin: BRK-Allianz.

Bürli, A. (2006). Internationale und vergleichende Heil- und Sonderpädagogik zwischen Naivität, Objektivismus und Skeptizismus. In F. Albrecht, A. Bürli, \& A. Erdélyi (Hrsg.), Aspekte internationaler Heil- und Sonderpädagogik (S. 25-46). Bad Heilbrunn: Klinkhardt.

Bürli, A., Strasser, U., \& Stein, A. (Hrsg.) (2009). Integration/Inklusion aus internationaler Sicht. Bad Heilbrunn: Klinkhardt.

D’Alessio, S., \& Watkins, A. (2009). International Comparisons of Inclusive Policy and Practices: Are we talking about the same thing? Research in Comparative and International Education, 4(3), 233-249.

Degener, T. (2009). Die UN-Behindertenrechtskonvention als Inklusionsmotor. Recht der Jugend und des Bildungswesens, 57, 200-219.

Deutscher Bundestag. (Hrsg.) (2008). Von Ausgrenzung zu Gleichberechtigung. Verwirklichung der Rechte von Menschen mit Behinderungen. Ein Handbuch für Abgeordnete zu dem Übereinkommen über die Rechte von Menschen mit Behinderungen und seinem Fakultativprotokoll (Deutsche Übersetzung des Handbuches der Vereinten Nationen und der Interparlamentarischen Union). Berlin: Deutscher Bundestag.

Deutsche UNESCO-Kommission e. V. (2014). Bonner Erklärung zur inklusiven Bildung in Deutschland. (verabschiedet von den Teilnehmenden des Gipfels „Inklusion - Die Zukunft der Bildung“ am 20. März 2014 in Bonn.). http://www.unesco.de/bonner_erklaerung.html. Zugegriffen: 16. Aug. 2014.

Dietze, T. (2013). Integration von Schülern mit sonderpädagogischem Förderbedarf in der Grundschule zur Situation in den 16 Bundesländern. Zeitschrift für Grundschulforschung, 6(1), 34-44.

Dorrance, C., \& Dannenbeck, C. (Hrsg.) (2013). Doing Inclusion: Inklusion in einer nicht inklusiven Gesellschaft. Bad Heilbrunn: Klinkhardt. 
Egilson, S., \& Traustadottir, R. (2009). Assistance to Pupils with Physical Disabilities in Regular Schools: Promoting Inclusion or Creating Dependency? European Journal of Special Needs Education, 24(1), 21-36.

European Agency for Development in Special Needs Education (2011). Mapping the Implementation of Policy for Inclusive Education. Odense: EADSNE.

European Agency for Development in Special Needs Education (2012). Special Needs Education Country Data 2012. Odense: EADSNE.

European Agency for Special Needs and Inclusive Education (2014). Country Information. Odense: EASNIE. http://www.european-agency.org/country-information. Zugegriffen: 16. Aug. 2014.

European Commission. (2014) Report on the implementation of the UN Convention on the Rights of Persons with Disabilities (CRPD) by the European Union. SWD (2014) 182 final. Brussels: European Commssion.

Eurydice (2014). Eurypedia. European Encyclopedia on National Education Systems. https://webgate. ec.europa.eu/fpfis/mwikis/eurydice/index.php?title=Countries. Zugegriffen: 28. März 2014.

Füssel, H.-P., \& Kretschmann, R. (1993). Gemeinsamer Unterricht für behinderte und nicht-behinderte Kinder. Witterschlick: Wehle.

Hans, M., \& Ginnold, A. (Hrsg.) (2000). Integration von Menschen mit Behinderung: Entwicklungen in Europa. Neuwied: Luchterhand.

Icelandic Human Rights Centre (2014). Submission to the Universal Periodic review of Iceland 12th UPR Session October 2011. http://www.humanrights.is/english/. Zugegriffen: 10. Sept. 2014.

Johnson, M. (2013). Schulische Inklusion in den USA - ein Lehrbeispiel für Deutschland? Bad Heilbrunn: Klinkhardt.

Klauer, K.J., \& Mitter, W. (Hrsg.) (1987). Vergleichende Sonderpädagogik. Berlin: Marhold.

Klemm, K. (2013). Inklusion in Deutschland - eine bildungsstatistische Analyse. Gütersloh: Bertelsmann Stiftung.

KMK (2011) = Kultusministerkonferenz. (2011). Inklusive Bildung von Kindern und Jugendlichen mit Behinderungen in Schulen (Beschuss der Kultusministerkonferenz vom 20.10.2011). Bonn: Ständige Konferenz der Kultusminister der Länder in der Bundesrepublik Deutschland.

KMK (2012) = Kultusministerkonferenz. (2012). Sonderpädagogische Förderung in Schulen 2001 bis 2010 (Statistische Veröffentlichungen Dokumentation Nr. 196- Februar 2012). Bonn: Ständige Konferenz der Kultusminister der Länder in der Bundesrepublik Deutschland.

Koch, S., \& Schemmann, M. (Hrsg.). (2009). Neoinstitutionalismus in der Erziehungswissenschaft. Wiesbaden: Springer VS.

Köpfer, A. (2013). Inclusion in Canada. Bad Heilbrunn: Klinkhardt.

Kottmann, B. (2006). Selektion in die Sonderschule. Bad Heilbrunn: Klinkhardt.

Kriwet, I. (2006). Widerspruchsvolle Sonderpädagogik - zur Integrations-/Inklusionsdiskussion in Schweden und Deutschland. In Albrecht, F., Bürli, A., \& Erdélyi, A. (Hrsg.), Internationale und vergleichende Heil- und Sonderpädagogik - Aktuelle Diskussionen, Ergebnisse und Herausforderungen (S. 189-198). Bad Heilbrunn: Klinkhardt.

Latham \& Watkins (2009). Völkerrechtliche Fragen des inklusiven Unterrichts in Deutschland im Zusammenhang mit dem Inkrafttreten des Übereinkommens der Vereinten Nationen über die Rechte von Menschen mit Behinderungen (Gutachten erstellt durch Latham \& Watkins für die Bundesarbeitsgemeinschaft Gemeinsam leben - gemeinsam lernen e. V.).

Limbach-Reich, A., \& Powell, J.J.W. (im Druck). Schüler/innen mit besonderem Förderungsbedarf im luxemburgischen Bildungssystem (Bildungsbericht Luxemburg). Luxemburg: Bildungsministerium/ Universität Luxemburg.

Löser, J.M., \& Werning, R. (2013): Inklusion aus internationaler Perspektive - ein Forschungsüberblick. Zeitschrift für Grundschulforschung, 6(1), 21-33.

Meijer, C.J.W. (2010). Special Needs Education in Europe: Inclusive Policies and Practices. Zeitschrift für Inklusion, 2010(2). http://www.inklusion-online.net/index.php/inklusion/article/view/56/60. Zugegriffen: 21. Nov. 2013.

Meyer, H.-D., \& Rowan, B. (Hrsg.) (2006). The New Institutionalism in Education. Albany: SUNY Press.

Mißling, S., \& Ückert, O. (2014). Vorabfassung der Studie Inklusive Bildung: Schulgesetze auf dem Prüfstand. Berlin: Deutsches Institut für Menschenrechte.

Moser, V. (Hrsg.) (2012). Die inklusive Schule: Standards für die Umsetzung. Stuttgart: Kohlhammer.

OHCHR (2014) = Office of the High Commissioner für Human Rights. (2014). Committee on the Rights of Persons with Disabilities. http://www.ohchr.org/en/hrbodies/crpd/pages/crpdindex.aspx. Zugegriffen: 02. April 2014. 
Paulsson, K., Nygren, G., Lübbeke, J., \& Lelgemann, R. (2011). Zur aktuellen Situation körperbehinderter Schülerinnen und Schüler in Schweden. Zeitschrift für Inklusion, 2011(4). http://www.inklusion-online.net/index.php/inklusion-online/article/view/77/77. Zugegriffen: 08. April 2014.

Pfahl, L. (2011). Techniken der Behinderung. Der deutsche Lernbehinderungsdiskurs, die Sonderschule und ihre Auswirkungen auf Bildungsbiografien. Bielefeld: transcript Verlag.

Pfahl, L. (2012). Bildung, Behinderung und Agency. Eine wissenssoziologische Untersuchung der Folgen schulischer Segregation und Inklusion. In R. Becker \& H. Solga (Hrsg.), Soziologische Bildungsforschung (Kölner Zeitschrift für Soziologie und Sozialpsychologie: Sonderheft 52, S. 415-436). Wiesbaden: Springer VS.

Powell, J.J.W. (2006). Special Education and the Risk of Becoming Less Educated. European Societies, $8(4), 577-599$.

Powell, J.J.W. (2009). Von schulischer Exklusion zur Inklusion? Eine neoinstitutionalistische Analyse sonderpädagogischer Fördersysteme in Deutschland und den USA. In S. Koch \& M. Schemmann (Hrsg.), Neoinstitutionalismus in der Erziehungswissenschaft (S. 213-232). Wiesbaden: Springer VS.

Powell, J.J.W. (2011). Barriers to Inclusion: Special Education in the United States and Germany. Boulder: Paradigm Publishers.

Powell, J.J.W., \& Pfahl, L. (2012). Sonderpädagogische Fördersysteme. In U. Bauer, U. Bittlingmayer, \& A. Scherr (Hrsg.), Handbuch Bildungs- \& Erziehungssoziologie (S. 721-739). Wiesbaden: Springer VS.

Preuss-Lausitz, U. (2001). Gemeinsamer Unterricht Behinderter und Nichtbehinderter. Zeitschrift für Erziehungswissenschaft, 4(2), 209-224.

Preuss-Lausitz, U. (2011). Wir dürfen nicht so tun, als hätten wir in Regelschulen keine Heterogenität. In Bertelsmann Stiftung, Beauftragter der Bundesregierung für die Belange behinderter Menschen, Deutsche UNESCO-Kommission und Sinn-Stiftung (Hrsg.), Gemeinsam lernen - Auf dem Weg zu einer inklusiven Schule (S. 46-50). Gütersloh: Bertelsmann Stiftung.

Preuss-Lausitz, U. (2014). Wissenschaftliche Begleitungen der Wege zur inklusiven Schulentwicklung in den Bundesländern. Versuch einer Übersicht. Berlin: Autor.

Ratzki, A. (2010). Skandinavische Bildungssysteme - Schule in Deutschland. Ein provokanter Vergleich. In G. Auernheimer (Hrsg.), Schieflagen im Bildungssystem (S. 23-31). Wiesbaden: Springer VS.

Richardson, J.G., \& Powell, J.J.W. (2011). Comparing Special Education: Origins to Contemporary Paradoxes. Stanford: Stanford University Press.

Schöler, J., Merz-Atalik, K., \& Dorrance, C. (2010). Auf dem Weg zur Schule für alle? Die Umsetzung der UN-Behindertenrechtskonvention im Bildungsbereich. München: Friedrich-Ebert-Stiftung.

Scott, W.R. (2008). Institutions and Organizations. Thousand Oaks: Sage.

Schnell, I. (2003). Geschichte schulischer Integration. Weinheim: Juventa.

Schumann, B. (2010). Inklusive Bildung in den nordischen Ländern im Kontext gesellschaftlicher Entwicklung. Zeitschrift für Inklusion, 2010(2). http://www.inklusion-online.net/index.php/inklusiononline/article/view/138/138. Zugegriffen: 02. April 2014.

Siehr, A., \& Wrase, M. (2014). Das Recht auf inklusive Schulbildung als Strukturfrage des deutschen Schulrechts - Anforderungen aus Art. 24 BRK und Art. 3 Abschn. 3 S. 2 GG. Recht der Jugend und des Bildungswesens, 2/2014, 161-182.

Sigurðardóttir, A.K., Guðjónsdóttir, H., \& Karlsdóttir, J. (2014). The Development of a School for All in Iceland: Equality, Threats and Political Conditions. In Blossing, U., Imsen, G., \& Moos, L. (Hrsg.), The Nordic Education Model (S. 95-113). Heidelberg: Springer.

Swedish Institute. (2014). Education in Sweden. http://sweden.se/society/education-in-sweden/start\#. Zugegriffen: 02. April 2014.

UNESCO (2008) = United Nations Educational, Scientific and Cultural Organization. (2008). Iceland. Presentation to the Workshop on Inclusive Education (6-7 March 2008, Helsinki). Paris: UNESCO.

United Nations (2013). Thematic study on the right of persons with disabilities to education. Report of the Office of the United Nations High Commissioner for Human Rights. United Nations, General Assembly, Human Rights Council. A/HRC/25/29.

United Nations (2014a). Convention and Optional Protocol Signatures and Ratifications. http://www. un.org/disabilities/countries.asp?navid=12\&pid=166. Zugegriffen: 15. Aug. 2014.

United Nations (2014b). Ad Hoc Committee on a Comprehensive and Integral International Convention on the Protection and Promotion of the Rights and Dignity of Persons with Disabilities. http://www. un.org/disabilities/default.asp?id=1423. Zugegriffen: 15. Aug. 2014. 
Weindling, P. (1999). International Eugenics: Swedish Sterilization in Context. Scandinavian Journal of History, 24(2), 179-197.

Werning, R. (2010). Inklusion zwischen Innovation und Überforderung. Zeitschrift für Heilpädagogik, 2010(8), 284-291.

Winzer, M., \& Mazurek, K. (2011). Analyzing Inclusive Schooling for Students with Disabilities in International Contexts. Journal of International Special Needs Education, 15(1), 12-23. 